\title{
Scrotal trauma: A case report and review of the literature
}

\author{
Harkanwal Randhawa, MD; Udi Blankstein, MD, MSc; Timothy Davies, MD, FRCSC
}

Department of Surgery, Division of Urology, McMaster University, Hamilton, ON, Canada

Cite as: Can Urol Assoc J 2019;13(6Supp14):S67-71. http://dx.doi.org/10.5489/cuaj.5981

\section{Case}

A healthy 25-year-old male with no medical history was evaluated by the urology service in the emergency department (ED) urgently after presenting with a chief complaint of scrotal swelling and pain. He described that he had been playing a game of baseball and was injured by direct contact between the ball and his scrotum, which immediately led to severe pain. The patient had initially deferred medical assessment, but presented to triage several hours after the initial trauma because of ongoing pain and swelling.

During initial assessment, the patient was clinically stable. He denied hematuria or any other voiding symptoms. Physical examination revealed significant edema and erythema along the right hemi-scrotum. The left hemi-scrotum was unremarkable. No lacerations suggesting penetrating trauma were noted.

Ultrasonography was ordered by the ED physician prior to urology consultation (Fig. 1). The radiology report revealed abnormal morphology of the right testicle, with disruption of the tunica albuginea (ruptured capsule). Additionally, there was concern for significant hematoma and extruded testicular components. After discussing the likely diagnosis of ruptured testicle, it was decided that scrotal exploration and repair or potential orchiectomy would be the best course of action, and as such, informed consent was obtained and the patient was prepared for the operating room.

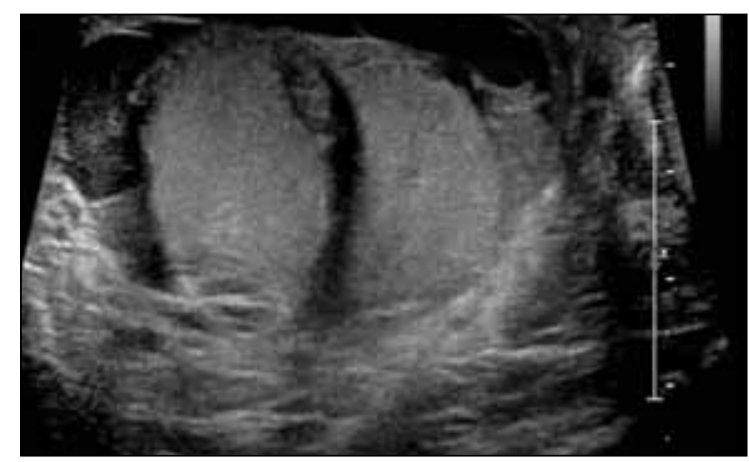

Fig. 1. Initial diagnostic scrotal ultrasonography of the right testicle demonstrating testicular rupture.

\section{Introduction}

Genitourinary (GU) injury is present in approximately $10 \%$ of cases of abdominal trauma. ${ }^{1}$ Of those GU injuries, up to $67 \%$ involve the external genitalia..$^{2,3} \mathrm{GU}$ trauma is more common in males, especially in the instance of genital injury. This increased incidence in males is secondary to both anatomical considerations and increased participation in activities such as contact sports, violent interaction, and war activities. ${ }^{2}$ The lower urinary tract is susceptible to traumatic injury by way of various mechanisms. These injuries, and their sequelae, can affect patients of any age or back- ground. Though lower urinary tract trauma rarely results in life-threating pathology, appropriate management of these injuries is pivotal in decreasing long-term morbidity. ${ }^{4}$

Although the external genitalia in males are at high risk of injury in trauma because of their extracorporeal location, the scrotum and testes are relatively well-protected from severe damage for the following reasons: 1 ) the testes are inherently mobile within the scrotum; 2) the scrotal skin provides reasonable elasticity allowing for internal structures to slip away from the point of contact in blunt trauma; 3 ) the cremasteric reflex offers a protective reflex mechanism; and 4) the tunica albuginea serves as a tough fibrous physical defense with its tensile strength. ${ }^{4-6}$ Resultantly, the incidence 
of scrotal or testicular injury in a trauma activation is generally considered to be less than one percent. However, the associated morbidity to the patient (including psychological effects of testicular injury or loss) and health system costs associated with these injuries warrant special attention. ${ }^{5,7-9}$

In general, there are two broad domains to consider when evaluating scrotal trauma: blunt vs. penetrating injury. Historically, blunt injury has been thought to comprise the significant majority (i.e., $75-80 \%$ ) of genital trauma. ${ }^{2,4}$ A recent analysis of the National Trauma Data Bank in the U.S. described a more balanced distribution of penetrating vs. blunt scrotal trauma, with both representing nearly half of all injuries. ${ }^{5}$ However, this significant difference in reported mechanism may be attributable to input biases and geopolitical determinants (e.g., increased incidence of gunshot wound [penetrating] injuries in the U.S. compared to other nations).

The importance of categorizing these these types of injuries into broad classes lies in the special diagnostic and management considerations for each type of injury (Fig. 2). For example, while just $1.5 \%$ of blunt testicular injury involves the gonads bilaterally, approximately $30 \%$ of penetrating scrotal injury will involve both testes. Additionally, penetrating injury occurs with associated injuries in approximately $70 \%$ of patients, decreasing the threshold of suspicion of damage to neighboring structures, such as the penis, bladder, urethra, and femoral vessels. ${ }^{2,4}$ However, workup for associated injury should not be neglected in the setting of blunt trauma either, and nearby structures should be appropriately investigated when clinical suspicion is present (e.g., blood at the urethral meatus suggesting urethral injury). ${ }^{4}$ Other domains of scrotal trauma, including thermal and degloving/scrotal wall avulsion injuries, are described in the literature as well and will be discussed here briefly.

There are several classifications applicable to trauma scenarios, including GU injury, which describe the severity and features associated with the presenting pathology. The most commonly used classification is the American Association

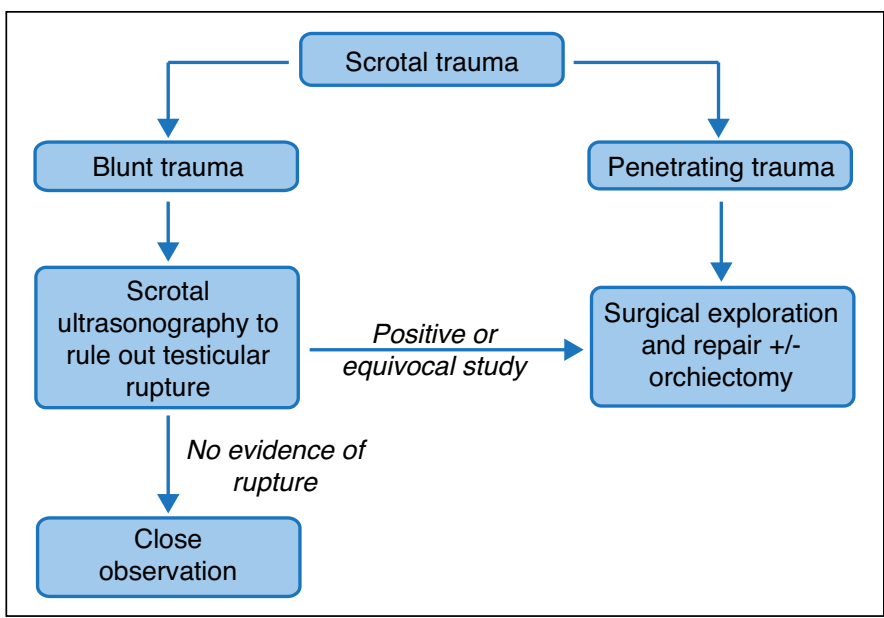

Fig. 2. Simplified diagnostic and management pathway in blunt and penetrating scrotal trauma. for the Surgery of Trauma's (AAST) injury scoring scale. ${ }^{2}$ The AAST provides an injury scale for both the testis and scrotum, each with a range of Grades I through $\mathrm{V}$, available publicly through the AAST at $h$ ttp://www.aast.org. ${ }^{10}$

A general principle for scrotal trauma, especially with severe injury (e.g., testicular rupture or fracture), is that early operative exploration and repair is the standard of care. ${ }^{7}$ Several case series have shown benefit to early exploration, including decreased rates of delayed orchiectomy, quicker resolution of pain, control of bleeding, theoretical concern of anti-sperm antibody production, preservation of spermatogenesis, shorter length of stay in hospital, and earlier return to activity. ${ }^{7,11-13}$

\section{Diagnostic evaluation}

A history of presenting illness, physical examination for evaluation of both intra- and extra-scrotal pathology, including peri-testicular and testicular masses, and simultaneous trauma evaluation (e.g., following standardized trauma guidelines) are paramount in any instance of scrotal trauma. Other important adjunct investigations, such as laboratory investigations (e.g., complete blood count) may also guide management, especially in the resuscitative phase of treatment.

Unfortunately, clinical examination of the scrotum in the acute post-trauma setting can be difficult and unreliable for a multitude of reasons (e.g., uncooperative patient because of pain, significant soft tissue swelling). ${ }^{8}$ The inability to elicit a reliable physical examination may result in incorrect triaging of patients for surgical exploration. Scrotal ultrasound can reliably assess scrotal injuries and diagnose testicular rupture with a high level of accuracy. High-frequency ultrasound with Doppler flow technique remains the imaging modality of choice for genital trauma. Ultrasound is used to assess the integrity of the affected tissue in scrotal trauma, and it is both readily available and inexpensive. ${ }^{14}$ Additionally, it can provide information regarding vascular perfusion of the testis, which is a critical determinant of the need for surgical management. ${ }^{15}$ For these reasons, ultrasound is also useful in the followup management of patients after scrotal trauma. This is especially true with specific injuries, such as hematoma formation, where small-sized hematomas without evidence of testicular rupture may be conservatively managed, but remain at high risk of infection and necrosis without resolution (incidence of up to $40 \%$ ), and thus require close followup with re-imaging. ${ }^{6}$

While sonography remains the primary imaging modality for scrotal pathology in trauma, there are limitations with this type of study, including the operator-dependent reliability of ultrasound and the availability of only a small field of view per image for the interpreting radiologist. Magnetic resonance imaging (MRI) has been well-established in the literature as a reliable modality for imaging in scrotal disease. Additionally, there is promising, emerging evidence to demonstrate its utility in the acute clinical setting, ${ }^{16}$ but 


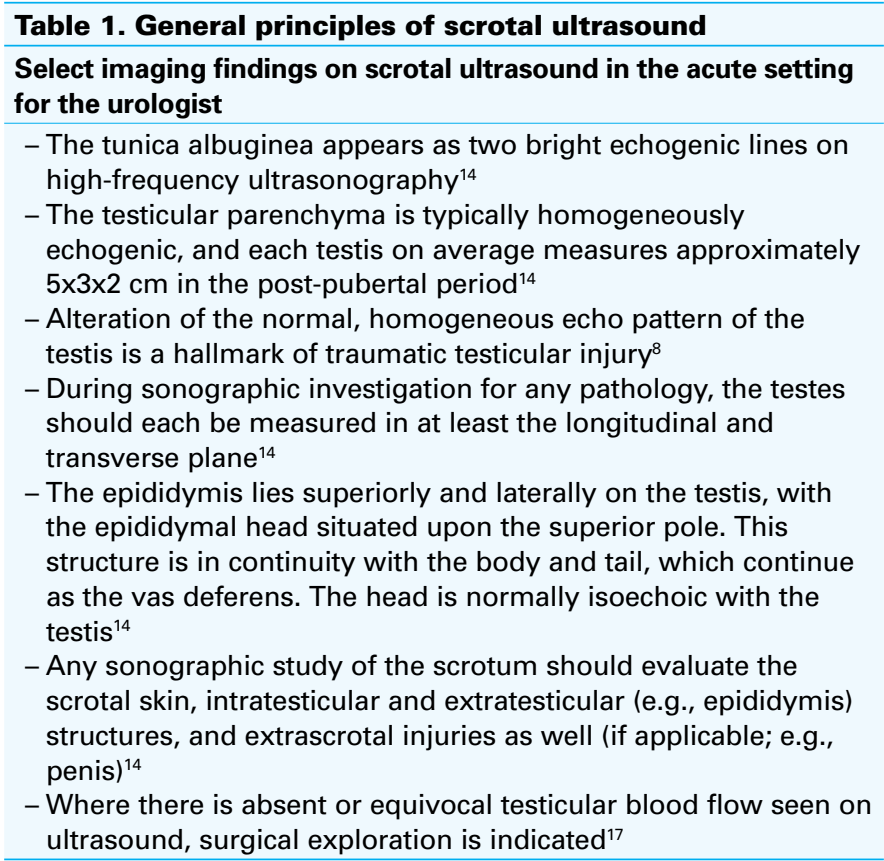

both the paucity of research in this context and the relative increased cost and decreased availability of this modality all pose significant barriers to its employment as the primary imaging tool in scrotal trauma.

Some of the general principles of scrotal ultrasonography are listed in Table 1. These findings are not meant to serve as diagnostic criteria for scrotal pathology on ultrasound, but rather provide a basis for interpreting key findings for the urologist in the acute clinical setting. Of note, it is important for the clinician to keep in mind that even in the setting of negative ultrasound findings, in patients where there is high risk or a high degree of suspicion for significant testicular injury (e.g., high-energy mechanism), early surgical exploration may be warranted. ${ }^{6}$

\section{Management considerations in scrotal trauma}

Early exploration with repair of testicular trauma is associated with increased rates of salvageability and preservation of fertility and hormonal function. Additionally, early exploration confers a reduced length of convalescence and disability, and may aid patients with faster return to activity. The objectives of management in operative cases of scrotal trauma, including exploration and repair, are testicular salvage, hemostasis, prevention of infection, and improved recovery time. Of note, for operative planning, a transverse scrotal incision is preferable in most cases. ${ }^{4}$

\section{Blunt}

Most genital injuries, in both males and females, are a result of blunt trauma. ${ }^{2}$ Sporting activities account for the majority of cases $(>50 \%)$ in which testicular injury occurs, and motor vehicle/bicycle injuries similarly account for a significant proportion of these traumas (approximately 20\%), ${ }^{6,8}$

\section{Testicular rupture/fracture}

Testicular rupture and/or fracture is present in approximately half of all cases of direct blunt scrotal trauma where dramatic compressive forces applied to the testis result in rupture of the fibrous tunica albuginea. Associated clinical signs and symptoms include sudden pain, nausea and emesis, and a tender, edematous, ecchymotic hemi-scrotum. ${ }^{2}$ Up to $90 \%$ of ruptured testes can be salvaged with surgical repair within 72 hours of injury. ${ }^{4,8,14}$ Typically, these injuries present unilaterally, with evidence of bilateral insult in approximately $1.5 \%$ of cases. ${ }^{14}$

On ultrasound, common findings of testicular rupture include discontinuity of the tunica albuginea and resultant abnormal testicular contour, heterogeneous echogenicity within the testis secondary to injury of the parenchyma, and regions of avascularity secondary to disruption of the tunica vasculosa. ${ }^{6,14}$ The overall sensitivity of scrotal ultrasonography for detection of traumatic testis rupture has been reported to be $64-100 \%$, with specificity from $65-93.5 \%$. In testicular fracture, a visible fracture line is a rare finding, present in less than $20 \%$ of cases. The presence of vascularity within the parenchyma is indicative of testicular salvageability. ${ }^{6,8,14}$

The current European Association of Urology guidelines recommend surgical exploration in all confirmed cases of testicular rupture and in patients with equivocal findings on imaging. ${ }^{2}$ Scrotal exploration includes either transverse or median raphe incision. After opening the tunica vaginalis, the hematoma should be evacuated and the testicle inspected for injury and viability. Any non-viable tissue should be debrided/ excised at this point, and any defect in the tunica albuginea should be closed with small, absorbable sutures to prevent the extruding force of progressive swelling on the seminiferous tubules. When primary closure is not possible or compromised, a flap or graft of tunica vaginalis may be used to repair the salvageable testis. Finally, although every attempt to salvage the testis should be performed, if the testicle itself is no longer viable, a simple orchiectomy should be performed. ${ }^{4,8}$

\section{Testicular dislocation}

Dislocation of the testis is a rare occurrence and is most common in motor vehicle accidents. Although uncommon, bilateral testicular dislocation may be seen in up to $25 \%$ of these cases. Dislocation may involve displacement within the subcutaneous/epifascial tissues, or internal dislocation into the external inguinal ring or inguinal canal. Treatment of dislocation involves manual reduction and orchidopexy. ${ }^{2,4}$ 


\section{Case followup}

The patient described in our case was promptly taken to the operating room for scrotal exploration. Intraoperatively, a significant hematoma was removed and the right testicle displayed a clear rupture of the tunica albuginea. As it was clear that the testicle otherwise appeared viable, it was decided that a primary repair would be attempted. This was done using 4-0 PDS suture. The testicle was then placed in the correct orientation into a sub-dartos pouch and the incision was closed primarily.

The patient was discharged on the first postoperative day without any complications and with a requisition for repeat ultrasonography in three months. Followup review in clinic revealed complete resolution of pain and normal morphology on physical examination. Repeat imaging showed only a minimal amount of volume loss to the right testicle, with anticipated post-surgical changes (Fig. 3).

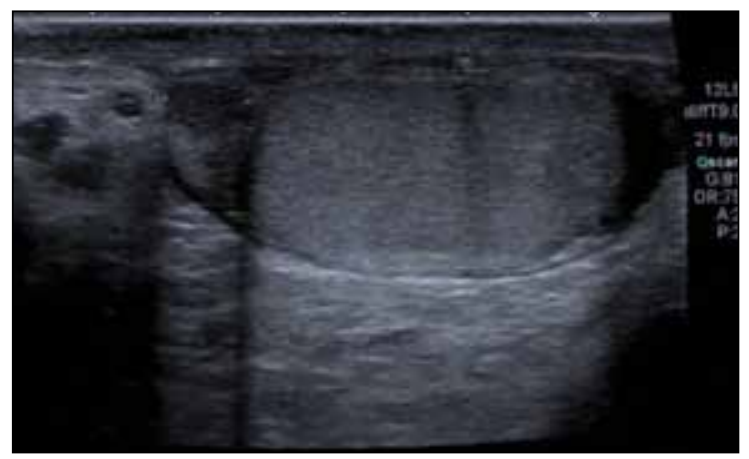

Fig. 3. Followup scrotal ultrasonography of the right testicle demonstrating minimal volume loss postoperatively.

\section{Scrotal hematoma or hematocele}

Hematoma and/or hematocele are the most common findings in the scrotum after blunt trauma. On scrotal ultrasonography, acute hematomas may appear isoechoic with the surrounding testis. Re-examination within 12-24 hours is helpful to assess for changes in echogenicity, as evolving hematomas may transform from hypoechoic to anechoic. Similarly, an acute hematocele typically appears echogenic vs. chronic hematocele, which is more anechoic and may reveal septations, loculations, and calcification. Hematomas may also be confined to the scrotal wall, where they sometimes appear as focal-thickening or intramural fluid collections. Finally, evaluation of blood flow of surrounding vessels to the testes is critical to assess for compressive forces when determining the need for surgical exploration. ${ }^{14}$

General management considerations include evacuation of the hematoma at the time of operative management, as well as excellent hemostasis to decrease the likelihood of re-bleed postoperatively. Current evidence promotes conservative management for hematoceles less than three times the size of the contralateral testis, or hematomas that are $<5 \mathrm{~cm}$ and non-expanding. This may involve treatment with scrotal support and ice. $2,11,12,17$

Patients with large hematoceles benefit from early operative management with evacuation of hematoma, even in the non-ruptured testis; this is due to the high rates of delayed exploration (up to $40 \%$ ) and orchiectomy (up to $15 \%$ ), infection, pain, atrophy, pressure necrosis, and prolonged length of stay in conservatively managed cases of large hematocele. ${ }^{2,11,12}$

\section{Penetrating}

Penetrating injuries require surgical exploration and subsequent debridement of any non-viable tissue. ${ }^{2,4}$ At the time of exploration, careful attention should be paid to structures within the spermatic cord, including the vas deferens and nearby vessels. The principles of management here are the same as in blunt trauma, with the primary goals of gonadal salvage and hemostasis in mind. ${ }^{4}$ Notably, penetrating testicular trauma does have a significantly lower testicular salvage rate (rates vary from $32-65 \%$ based on historical case series). ${ }^{4}$

\section{Gunshot wounds}

Most penetrating injuries to the external genitalia are secondary to gunshots. ${ }^{4}$ Information about the offending weapon and mechanism of injury when scrotal trauma is secondary to a gunshot wound is helpful in estimating the amount of energy transfer and subsequent degree of injury. This includes information regarding the range, caliber, and type of weapon. ${ }^{2}$

Notably, the vas deferens is injured in approximately $7-9 \%$ of gunshot wounds to the scrotum. When vas injury is noted, ligation with non-absorbable suture should be performed. Finally, an important consideration in this scenario is that approximately $30 \%$ of gunshot wounds cause trauma to both testes and exploration of the contralateral testis may be indicated. Principles of management with gunshot wounds include urgent exploration, irrigation of tissues involved, excision of foreign bodies, antibiotic prophylaxis, and wound closure. ${ }^{4}$

\section{Bites}

Bites causing injury to the genitalia are rare, and more commonly are a result of animals (especially dogs) than humans. Bite wounds are typically minor traumas, but an 
important consideration is appropriate antimicrobial prophylaxis because of the burden of bacterial transfer with this mechanism of injury. Initial management of bites includes irrigation, debridement, and antibiotic prophylaxis. Closure of the wound in the case of dog bites can be performed primarily. However, human bites are more likely to produce a contaminated wound, warranting healing by secondary intention in most cases. ${ }^{4}$ These bites have the potential to introduce polymicrobial oral microflora, which includes both aerobic and anaerobic pathogens. ${ }^{18}$

For animal bites, first-line antibiotic prophylaxis is with amoxicillin-clavulanic acid, a second-generation cephalosporin with anaerobic coverage (e.g., cefoxitin, cefotetan), or clindamycin in combination with a fluoroquinolone. Additionally, depending on geographic location and animal(s) involved, the need for rabies vaccination must be considered. For human bites, transmission of viral pathogens is a key consideration (e.g., hepatitis $B$, human immunodeficiency virus). In all cases, tetanus vaccination should be considered. Antibiotic prophylaxis, or empiric treatment, is warranted with either amoxicillin-clavulanic acid or moxifloxacin.2,4 Additionally, the clinician must keep in mind the increased likelihood of delayed presentation with human bites because of embarrassment or attempts at self-treatment initially. ${ }^{4,18}$ This may contribute to worse infection of the scrotum at initial presentation.

\section{Thermal}

Genital burns are rarely seen as isolated injuries. As such, important management considerations include principles of burn treatment, such as complete physical examination with calculation of total body surface area involved, fluid resuscitation, and tetanus and antibiotic prophylaxis. ${ }^{19}$ These injuries are typically secondary to industrial flames or chemical insult. All genital burn injuries are treated conservatively unless there is evidence of full-thickness burn. ${ }^{2}$ These injuries, which are insensate and have a dry, charred appearance, may impair functioning of the testis by affecting spermatogenesis. ${ }^{6,8}$ Thirddegree burns typically require operative management by way of excision and reconstruction. ${ }^{8}$

\section{Degloving/avulsion}

Avulsion injuries resulting in skin loss are typically a result of rapid deceleration mechanisms. In this scenario, the elastic skin of the genitalia is entangled in clothing caught on a stationary object and forcefully sheared as a result (e.g., motor vehicle accidents). ${ }^{8}$ Minor avulsions are more common, and typically the skin and superficial dartos fascia are the only involved layers. These injuries may be managed as simple lacerations with irrigation and primary two-layer closure of the deep fascia and skin, with an interlocking, running, absorbable suture. These repairs should be performed urgently to decrease bacterial colonization of deeper layers of the wound. Because of the elasticity, compliance, and redundancy of scrotal skin, defects with up to $60 \%$ skin loss may be closed primarily. More complex avulsion injuries should be managed with debridement and close observation prior to a delayed reconstruction (via primary re-approximation or skin graft), of which details are discussed elsewhere. ${ }^{19}$

Competing interests: Dr. Davies has been an advisory board member for Paladin; and has received grants/honoraria from Boston Scientific, Paladin, and Pfizer. The remaining authors report no competing personal or financial conflicts.

This paper has been peer reviewed.

\section{References}

1. Bryk DJ, Zhao LC. Guideline of guidelines: A review of urological trauma guidelines. BJU Int 2016;117:22634. https://doi.org/10.1111/bju.13040

2. Kitrey ND, Djakovic N, Gonsalves M, et al. EAU guidelines on urological trauma. European Association of Urology, 2016. Available at: https://uroweb.org/individual-guidelines/non-oncology-guidelines/. Accessed Feb. 7, 2019.

3. Morey AF, Brandes S, Dugi DD 3rd. Urotrauma: AUA guideline. J Urol 2014;192:327-35 https://doi. org/10.1016/i.juro.2014.05.004

4. Wein AJ, Kavoussi LR, Partin AW, et al. Campbell-Walsh Urology: 11th ed. US: Elsevier; 2016. P. 4176.

5. Grigorian A, Livingston JK, Schubl SD, et al. National analysis of testicular and scrotal trauma in the USA. Res Rep Urol 2018:10;51-6. https://doi.org/10.2147/RRU.S172848

6. Bhatt S, Dogra VS. Role of US in testicular and scrotal trauma. RadioGraphics 2008;28:1617-29. https://doi.org/10.1148/rg.286085507

7. Buckley JC, MCAninch JW. Use of ultrasonography for the diagnosis of testicular injuries in blunt scrotal trauma. J Urol 2006;175:175-8. https://doi.org/10.1016/S0022-5347(05)00048-0

8. Morey AF, Metro MJ, Carney KJ, et al. Consensus on genitourinary trauma: External genitalia. BJU Int 2004;94:507-15. https://doi.org/10.1111/j.1464-410X.2004.04993.x

9. McGeady JB, Breyer BN. Current epidemiology of genitourinary trauma. Urol Clin North Am 2013;40:32334. https://doi.org/10.1016/i.ucl.2013.04.001

10. Moore EE, Malangoni MA, Cogbill TH, et al. Organ injury scaling VIl: Cervical vascular, peripheral vascular, adrenal, penis, testis, and scrotum. J Irauma 1996;41:523-4. https://doi.org/10.1097/00005373199609000-00023

11. Cass AS, Luxenberg M. Value of early operation in blunt testicular contusion with hematocele. J Urol 1988;139:746-7. https://doi.org/10.1016/S0022-5347(17)42620-6

12. Cass AS. Testicular trauma. J Urol 1983;129:299-300. https://doi.org/10.1016/S00225347(17)52062-5

13. Corrales JG, Corbel L, Cipolla B, et al. Accuracy of ultrasound diagnosis after blunt testicular trauma. J Urol 1993;150:1834-6. https://doi.org/10.1016/S0022-5347(17)35909-8

14. Nicola R, Carson N, Dogra VS. Imaging of traumatic injuries to the scrotum and penis. A/R 2014;202:W51220. https://doi.org/10.2214/AJR.13.11676

15. Adlan T, Freeman SJ. Can ultrasound help to manage patients with scrotal trauma? Ultrasound 2014;22:205-12. https://doi.org/10.1177/1742271X14545911

16. Parenti GC, Feletti F, Carnevale A, et al. Imaging of the scrotum: Beyond sonography. Insights Imaging 2018;9:137-48. https://doi.org/10.1007/s13244-017-0592-z

17. Michaud JE, Di Carlo HN, Pierorazio PM. Diagnosis and management of the acute scrotum. AUA Update Series 2016;35:391-7.

18. $\mathrm{Ng} \mathrm{D}$, Chan T, Pothiawala S. A human bite on the scrotum: Case report and review of management in the emergency department. J Emerg Med 2018;54:537-9. https://doi.org/10.1016/i. jemermed.2017.12.039

19. Chang AJ, Brandes SB. Advances in diagnosis and management of genital injuries. Urol Clin N Am 2013;40:427-38. https://doi.org/10.1016/i.ucl.2013.04.013

Correspondence: Dr. Timothy Davies, Department of Surgery, Division of Urology, McMaster University, Hamilton, ON, Canada; tdavies@mcmaster.ca 